\title{
STABILITY OF HUMORAL IMMUNITY VIRUS INFECTION MODEL WITH GENERAL INCIDENCE RATE AND DISTRIBUTED DELAYS
}

\author{
A. M. ELAIW, A. ALHEJELAN, AND A. M. SHEHATA \\ Received 17 December, 2013
}

\begin{abstract}
In this paper, we investigate the global stability of a virus infection model with humoral immune response and distributed intracellular delays. The incidence rate of infection is given by general functional response. The model has two types of distributed time delays which describe the time needed for infection of uninfected cell and virus replication. Lyapunov functionals are constructed and LaSalle's invariance principle is used to establish the global asymptotic stability of all steady states of the model. We have proven that, if the basic reproduction number $R_{0}$ is less than or equal unity, then the uninfected steady state is globally asymptotically stable (GAS), and if the humoral immune response reproduction number $R_{1}$ is less than or equal unity and $R_{0}>1$, then the infected steady state without humoral immune response exists and it is GAS, and if $R_{1}>1$, then the infected steady state with humoral immune response exists and it is GAS. Numerical simulations have been carried out with a specific form of the incidence rate function. We have shown that, both the numerical and theoretical results are consistent.
\end{abstract}

2010 Mathematics Subject Classification: 34D20; 34D23; 37N25; 92D30

Keywords: virus dynamics, intracellular delay, global stability, humoral immunity, Lyapunov functional

\section{INTRODUCTION}

Recently, several mathematical models have been proposed and developed to understand the interaction of virus and target cells, such as human immunodeficiency virus (HIV) (see [3, 4, 6-11, 13, 15, 16, 23, 25, 26, 29, 31,32]), hepatitis B virus (HBV) [21, 28,33], hepatitis C virus (HCV) [27] and [38] and human T cell leukemia HTLV [22], etc. Mathematical modeling and model analysis of the viral infection process are helpful for estimating key parameter values, and guiding development efficient anti-viral drug therapies. Immunity is a biological term that describes a state of having sufficient biological defenses to avoid infection, disease or other unwanted biological invasion. Humoral immunity is the aspect of immunity that is mediated by secreted antibodies. In malaria infection, the humoral immunity is more effective than cell-mediated immunity [5]. Mathematical models for virus dynamics with humoral immune response have been developed in [1, 2, 12, 14, 19, 24, 30, 34, 36]. 
The basic virus dynamics model with humoral immune response was introduced by Murase et. al. [24] as:

$$
\begin{aligned}
& \dot{x}(t)=\lambda-d x(t)-\beta x(t) v(t), \\
& \dot{y}(t)=\beta x(t) v(t)-a y(t), \\
& \dot{v}(t)=N a y(t)-c v(t)-q v(t) z(t), \\
& \dot{z}(t)=\operatorname{rv}(t) z(t)-\mu z(t),
\end{aligned}
$$

where $x(t), y(t), v(t)$ and $z(t)$ represent the populations of uninfected cells, infected cells, virus and B cells at time $t$, respectively; $\lambda$ is the rate at which new uninfected cells are generated from the body; $d$ is the natural death rate constant of uninfected cells; $\beta$ is the infection rate constant; $N$ is the number of free viruses produced during the average infected cell life span; $a$ is the death rate constant of infected cells; $c$ is the clearance rate constant of the virus particles; $r$ and $\mu$ are the proliferation rate and death rate constants of B cells; $q$ is the B cells neutralize rate. Note that the forenamed system (1.1)-(1.4) does not contain an intracellular time delay between the infection of a cell and the production of new virus particles, despite the fact that intracellular delay actually exists (see e.g. [4, 6, 7, 9-11,13,15, 16, 23]). Further, the infection rate is assumed to be bilinear in $x$ and $v$, but many researches suggested that the bilinear incidence rate associated with the mass action principle is insufficient to describe the infection process in detail (see e.g. [18, 20]). Thus, it is reasonable to assume that the infection rate is given by nonlinear incidence rate. The incidence rate has been considered in the viral infection models with humoral immune response by different forms as: saturated incidence rate, $\frac{\beta x v}{1+\alpha v}$ where $\alpha \geq 0$ [12,36], Beddington-DeAngelis functional response, $\frac{\beta x v}{1+\gamma x+\alpha v}, \alpha, \gamma \geq 0$ [14,37], and general form, $\Phi(x, v) v$ [35]. In [35], model with discrete time delay has been investigated.

In this paper, we propose a virus infection model with humoral immune response and with general incidence rate. We incorporate two types of distributed delays into this model to account the time delay between the time that target cells are contacted by the virus particles and the time the emission of infectious (matures) virus particles. The global stability of this model is established using Lyapunov functionals and LaSalle's invariance principle. We prove that the global dynamics of this model is determined by the basic reproduction number $R_{0}$ and humoral immune response reproduction number $R_{1}$. If $R_{0} \leq 1$, then the uninfected steady state is globally asymptotically stable (GAS), if $R_{1} \leq 1<R_{0}$, then the infected steady state without humoral immune response exists and it is GAS, and if $R_{1}>1$, then the infected steady state with humoral response exists and it is GAS. Numerical simulations is carried out to confirm our theoretical results. 


\section{THE MODEL}

In this section, we propose a mathematical model of virus infection which describes the interaction of the virus with target cells, taking into account the humoral immune response.

$$
\begin{aligned}
\dot{x}(t) & =\lambda-d x(t)-\Phi(x(t), v(t)) v(t), \\
\dot{y}(t) & =\int_{0}^{h} f(\tau) e^{-m \tau} \Phi(x(t-\tau), v(t-\tau)) v(t-\tau) d \tau-a y(t), \\
\dot{v}(t) & =N a \int_{0}^{\omega} g(\tau) e^{-n \tau} y(t-\tau) d \tau-c v(t)-q v(t) z(t), \\
\dot{z}(t) & =r v(t) z(t)-\mu z(t) .
\end{aligned}
$$

All the variables and parameters of the model have the same meanings as given in section 1. To account for the time lag between viral contacting an uninfected cell and the production of new virus particles, two distributed intracellular delays are introduced. It assumed that the uninfected cells are contacted by the virus particles at time $t-\tau$ become infected cells at time $t$, where $\tau$ is a random variable with a probability distribution $f(\tau)$ over the interval $[0, h]$ and $h$ is limit superior of this delay. The factor $e^{-m \tau}$ account for the probability of surviving the time period of delay, where $m$ is the death rate of infected cells but not yet virus producer cells. On the other hand, it is assumed that, a cell infected at time $t-\tau$ starts to yield new infectious virus at time $t$ where $\tau$ is distributed according to a probability distribution $g(\tau)$ over the interval $[0, \omega]$ and $\omega$ is limit superior of this delay. The factor $e^{-n \tau}$ account for the probability of surviving the time period of delay, where $n$ is a constant. The incidence rate is given by a general function $\Phi(x, v) v$ where $\Phi \in C^{1}([0, \infty) \times$ $[0, \infty), \mathbb{R})$ and satisfies the following assumptions [35]:

Assumption A1. $\Phi(x, v)>0$ for all $x>0, v>0$, and $\Phi(0, v)=0$.

Assumption A2. $\frac{\partial \Phi(x, v)}{\partial x}>0$ for all $x>0$ and $v>0$.

Assumption A3. $\frac{\partial \Phi(x, v)}{\partial v}<0$ for all $x>0$ and $v>0$.

Assumption A4. $\frac{\partial(\Phi(x, v) v)}{\partial v}>0$ for all $x>0$ and $v>0$.

The probability distribution functions $f(\tau)$ and $g(\tau)$ are assumed to satisfy $f(\tau)>$ 0 and $g(\tau)>0$, and

$$
\int_{0}^{h} f(\tau) d \tau=\int_{0}^{\omega} g(\tau) d \tau=1, \quad \int_{0}^{h} f(u) e^{s u} d u<\infty, \quad \int_{0}^{\omega} g(u) e^{s u} d u<\infty
$$


where $s$ is a positive number. Then

$$
0<\int_{0}^{h} f(\tau) e^{-m \tau} d \tau \leq 1,0<\int_{0}^{\omega} g(\tau) e^{-n \tau} d \tau \leq 1, \quad m, n \geq 0 .
$$

Let us denote:

$$
F=\int_{0}^{h} f(\tau) e^{-m \tau} d \tau, \quad G=\int_{0}^{\omega} g(\tau) e^{-n \tau} d \tau .
$$

The initial conditions for system (2.1)-(2.4) take the form

$$
\begin{aligned}
x(\theta) & =\varphi_{1}(\theta), y(\theta)=\varphi_{2}(\theta), \\
v(\theta) & =\varphi_{3}(\theta), z(\theta)=\varphi_{4}(\theta), \\
\varphi_{j}(\theta) & \geq 0, \theta \in[-\rho, 0), \quad j=1, \ldots, 4, \\
\varphi_{j}(0) & >0, j=1, \ldots, 4,
\end{aligned}
$$

where $\rho=\max \{h, \omega\}, \quad\left(\varphi_{1}(\theta), \varphi_{2}(\theta), \varphi_{3}(\theta), \varphi_{4}(\theta)\right) \in C\left([-\rho, 0], \mathbb{R}_{+}^{4}\right)$, where $C\left([-\rho, 0], \mathbb{R}_{+}^{4}\right)$ is the Banach space of continuous functions mapping the interval $[-\rho, 0]$ into $\mathbb{R}_{+}^{4}$. By the fundamental theory of functional differential equations [17], system (2.1)-(2.4) has a unique solution satisfying the initial conditions (2.5).

\subsection{Non-negativity and boundedness of solutions}

In the following, we establish the non-negativity and boundedness of solutions of (2.1)-(2.4) with initial conditions (2.5).

Proposition 1. Assume that Assumption Al is satisfied. Let $(x(t), y(t), v(t), z(t))$ be any solution of (2.1)-(2.4) satisfying the initial conditions (2.5), then $x(t), y(t), v(t)$ and $z(t)$ are all non-negative for $t \geq 0$ and ultimately bounded.

Proof. First, we prove that $x(t)>0$ for all $t \geq 0$. Assume that $x(t)$ loses its nonnegativity on some local existence interval $[0, \ell]$ for some constant $\ell$ and let $t_{1} \in[0, \ell]$ be such that $x\left(t_{1}\right)=0$. From Eq. (2.1) we have $\dot{x}\left(t_{1}\right)=\lambda>0$. Hence, $x(t)>0$ for some $t \in\left(t_{1}, t_{1}+\varepsilon\right)$ where $\varepsilon>0$ is sufficiently small. This leads to contradiction and hence $x(t)>0$, for all $t \geq 0$. Now from Eqs. (2.2)-(2.4) we have

$$
\begin{aligned}
& y(t)=y(0) e^{-a t}+\int_{0}^{t} e^{-a(t-\eta)} \int_{0}^{h} f(\tau) e^{-m \tau} \Phi(x(\eta-\tau), v(\eta-\tau)) v(\eta-\tau) d \tau d \eta, \\
& v(t)=v(0) e^{-\int_{0}^{t}(c+q z(\xi)) d \xi}+N a \int_{0}^{t} e^{-\int_{\eta}^{t}(c+q z(\xi)) d \xi} \int_{0}^{\omega} g(\tau) e^{-n \tau} y(\eta-\tau) d \tau d \eta,
\end{aligned}
$$


$z(t)=z(0) e^{-\int_{0}^{t}(\mu-r v(\xi)) d \xi}$

confirming that $y(t) \geq 0, v(t) \geq 0$ and $z(t) \geq 0$ for all $t \in[0, \rho]$. By a recursive argument, we obtain $y(t) \geq 0, v(t) \geq 0$ and $z(t) \geq 0$ for all $t \geq 0$.

Next we show the boundedness of the solutions of the system. From Eq. (2.1) we have $\dot{x}(t) \leq \lambda-d x(t)$ and thus $\limsup _{t \rightarrow \infty} x(t) \leq \frac{\lambda}{d}$. Let $X_{1}(t)=\int_{0}^{h} f(\tau) e^{-m \tau}$ $x(t-\tau) d \tau+y(t)$, then

$$
\begin{aligned}
\dot{X}_{1}(t)= & \int_{0}^{h} f(\tau) e^{-m \tau}(\lambda-d x(t-\tau)-\Phi(x(t-\tau), v(t-\tau)) v(t-\tau)) d \tau \\
& +\int_{0}^{h} f(\tau) e^{-m \tau} \Phi(x(t-\tau), v(t-\tau)) v(t-\tau) d \tau-a y(t), \\
& =\lambda \int_{0}^{h} f(\tau) e^{-m \tau} d \tau-d \int_{0}^{h} f(\tau) e^{-m \tau} x(t-\tau) d \tau-a y(t) \\
\leq & \lambda \int_{0}^{h} f(\tau) e^{-m \tau} d \tau-\sigma_{1}\left[\int_{0}^{h} f(\tau) e^{-m \tau} x(t-\tau) d \tau+y(t)\right] \\
= & \lambda \int_{0}^{h} f(\tau) e^{-m \tau} d \tau-\sigma_{1} X_{1}(t) \leq \lambda-\sigma_{1} X_{1}(t),
\end{aligned}
$$

where $\sigma_{1}=\min \{d, a\}$. Hence $\limsup \sup _{t \rightarrow \infty} X_{1}(t) \leq L_{1}$, where $L_{1}=\frac{\lambda}{\sigma_{1}}$. Since $\int_{0}^{h} f(\tau) e^{-m \tau} x(t-\tau) d \tau>0$, then $\limsup _{t \rightarrow \infty} y(t) \leq L_{1}$. On the other hand, let $X_{2}(t)=v(t)+\frac{q}{r} z(t)$, then

$$
\begin{aligned}
\dot{X}_{2}(t) & =N a \int_{0}^{\omega} g(\tau) e^{-n \tau} y(t-\tau) d \tau-c v(t)-\frac{q \mu}{r} z(t) \\
& \leq N a L_{1} \int_{0}^{\omega} g(\tau) e^{-n \tau} d \tau-\sigma_{2}\left(v(t)+\frac{q}{r} z(t)\right)
\end{aligned}
$$




$$
=N a L_{1} \int_{0}^{\omega} g(\tau) e^{-n \tau} d \tau-\sigma_{2} X_{2}(t) \leq N a L_{1}-\sigma_{2} X_{2}(t),
$$

where $\sigma_{2}=\min \{c, \mu\}$. Hence $\limsup \sup _{t \rightarrow \infty} X_{2}(t) \leq L_{2}$, where $L_{2}=\frac{N a L_{1}}{\sigma_{2}}$. Since $v(t) \geq 0$ and $y(t) \geq 0$, then $\limsup _{t \rightarrow \infty} v(t) \leq L_{2}$ and $\limsup _{t \rightarrow \infty} z(t) \leq L_{3}$, where $L_{3}=\frac{r}{q} L_{2}$. Therefore, $x(t), y(t), v(t)$ and $z(t)$ are ultimately bounded.

\subsection{Steady states}

We define the basic reproduction number for system (2.1)-(2.4) as

$$
R_{0}=\frac{N F G \Phi\left(x_{0}, 0\right)}{c}
$$

where $x_{0}=\lambda / d$. To calculate the steady state, we let the right-hand side of Eqs. (2.1)-(2.4) be zero:

$$
\begin{aligned}
\lambda-d x-\Phi(x, v) v & =0, \\
F \Phi(x, v) v-a y & =0, \\
N a G y-c v-q v z & =0, \\
r v z-\mu z & =0 .
\end{aligned}
$$

From (2.9), either $z=0$ or $z \neq 0$. If $z=0$, then from (2.6)-(2.8) we get

$$
y=\frac{F(\lambda-d x)}{a}=\frac{c}{N a G} v, \quad v=\frac{N F G(\lambda-d x)}{c} .
$$

Substituting from (2.10) into (2.7) we get:

$$
\left[\Phi\left(x, \frac{N F G(\lambda-d x)}{c}\right)-\frac{c}{N F G}\right] v=0 .
$$

Eq. (2.11) has two possible solutions $v=0$ or $v \neq 0$. If $v=0$, then from Eqs. (2.6) and (2.7), we get $x=x_{0}$ and $y=0$ which leads to the uninfected steady state $E_{0}\left(x_{0}, 0,0,0\right)$. If $v \neq 0$, then we have

$$
\Phi\left(x, \frac{N F G(\lambda-d x)}{c}\right)-\frac{c}{N F G}=0 .
$$

Let

$$
M(x)=\Phi\left(x, \frac{N F G(\lambda-d x)}{c}\right)-\frac{c}{N F G} .
$$

From Assumptions $\mathrm{A} 1$ and $\mathrm{A} 2$, function $M(x)$ is strictly increasing with respect to $x$. Moreover, $M(0)=\Phi\left(0, \frac{N F G \lambda}{c}\right)-\frac{c}{N F G}=-\frac{c}{N F G}<0$. Also, $M\left(x_{0}\right)=$ $\Phi\left(x_{0}, 0\right)-\frac{c}{N F G}=\frac{c}{N F G}\left(R_{0}-1\right)$. Therefore, if $R_{0}>1$, then there exist a unique $x_{1} \in\left(0, x_{0}\right)$ such that $M\left(x_{1}\right)=0$. From Eq. (2.10) we obtain $y_{1}>0$ and $v_{1}>0$. 
It follows that, if $R_{0}>1$, then there is an infected steady state without immune response $E_{1}\left(x_{1}, y_{1}, v_{1}, 0\right)$.

The other possibility of Eq. (2.9) $z \neq 0$ leads to

$$
v_{2}=\frac{\mu}{r}, \quad y_{2}=\frac{F \Phi\left(x_{2}, v_{2}\right) v_{2}}{a}, \quad z_{2}=\frac{N F G \Phi\left(x_{2}, v_{2}\right)}{q}-\frac{c}{q} .
$$

Let

$$
M_{1}(x)=\lambda-d x-\Phi\left(x, v_{2}\right) v_{2} .
$$

From Assumptions $\mathrm{A} 1$ and $\mathrm{A} 2$, function $M_{1}$ is strictly decreasing with respect to $x$. We have also $M_{1}(0)=\lambda>0$ and $M_{1}\left(x_{0}\right)=-\Phi\left(x_{0}, v_{2}\right) v_{2}<0$. Thus, there exist a unique $x_{2} \in\left(0, x_{0}\right)$ such that $M_{1}\left(x_{2}\right)=0$. Now we are ready to define the humoral immune response reproduction number as:

$$
R_{1}=\frac{N F G \Phi\left(x_{2}, v_{2}\right)}{c}
$$

Hence, $z_{2}$ cab be rewritten as $z_{2}=\frac{c}{q}\left(R_{1}-1\right)$. It follows that, if $R_{1}>1$, then there is an infected steady state with immune response $E_{2}\left(x_{2}, y_{2}, v_{2}, z_{2}\right)$.

From above we have the following result.

Lemma 1. Assume that Assumptions A1 and A2 are satisfied and

(i) if $R_{0} \leq 1$, then there exists only one positive steady state $E_{0}$,

(ii) if $R_{0}>1$, then there exist two positive steady states $E_{0}$ and $E_{1}$, and

(iii) if $R_{1}>1$, then there exist three positive steady states $E_{0}, E_{1}$ and $E_{2}$.

\subsection{Global stability}

In this section, we prove the global stability of the steady states of system (2.1)(2.4) employing the method of Lyapunov functional and LaSalle's invariance principle. Next we shall use the following notation: $u=u(t)$, for any $u \in\{x, y, v, z\}$. Throughout the paper, we let

$$
H(u)=u-1-\ln u .
$$

where $H:(0, \infty) \rightarrow[0, \infty), H(u) \geq 0$ for any $u>0$ and $H$ has the global minimum $H(1)=0$.

Theorem 1. If Assumptions A1-A3 hold true and $R_{0} \leq 1$, then $E_{0}$ is GAS.

Proof. Define a Lyapunov functional $W_{0}$ as follows:

$$
\begin{aligned}
W_{0}= & N F G\left[x-x_{0}-\int_{x_{0}}^{x} \frac{\Phi\left(x_{0}, 0\right)}{\Phi(\eta, 0)} d \eta+\frac{1}{F} y\right. \\
& +\frac{1}{F} \int_{0}^{h} f(\tau) e^{-m \tau} \int_{0}^{\tau} \Phi(x(t-\theta), v(t-\theta)) v(t-\theta) d \theta d \tau
\end{aligned}
$$




$$
\left.+\frac{a}{F G} \int_{0}^{\omega} g(\tau) e^{-n \tau} \int_{0}^{\tau} y(t-\theta) d \theta d \tau\right]+v+\frac{q}{r} z .
$$

The time derivative of $W_{0}$ along the trajectories of (2.1)-(2.4) satisfies

$$
\begin{aligned}
\frac{d W_{0}}{d t}= & N F G\left[\left(1-\frac{\Phi\left(x_{0}, 0\right)}{\Phi(x, 0)}\right)(\lambda-d x-\Phi(x, v) v)\right. \\
& +\frac{1}{F} \int_{0}^{h} f(\tau) e^{-m \tau} \Phi(x(t-\tau), v(t-\tau)) v(t-\tau) d \tau-\frac{a}{F} y \\
& +\frac{1}{F} \int_{0}^{h} f(\tau) e^{-m \tau}(\Phi(x, v) v-\Phi(x(t-\tau), v(t-\tau)) v(t-\tau)) d \tau \\
& \left.+\frac{a}{F G} \int_{0}^{\omega} g(\tau) e^{-n \tau}(y-y(t-\tau)) d \tau\right] \\
& +N a \int_{0}^{\omega} g(\tau) e^{-n \tau} y(t-\tau) d \tau-c v-q v z+q v z-\frac{q \mu}{r} z, \\
= & \frac{N F G d}{\Phi(x, 0)}\left(x_{0}-x\right)\left(\Phi(x, 0)-\Phi\left(x_{0}, 0\right)\right) \\
& +\left(N F G \Phi(x, v) \frac{\Phi\left(x_{0}, 0\right)}{\Phi(x, 0)}-c\right) v-\frac{q \mu}{r} z, \\
= & \frac{N F G d}{\Phi(x, 0)}\left(x_{0}-x\right)\left(\Phi(x, 0)-\Phi\left(x_{0}, 0\right)\right)+c\left(R_{0} \frac{\Phi(x, v)}{\Phi(x, 0)}-1\right) v-\frac{q \mu}{r} z .
\end{aligned}
$$

From Assumptions A2-A3 we have

$$
\begin{gathered}
\left(x_{0}-x\right)\left(\Phi(x, 0)-\Phi\left(x_{0}, 0\right)\right) \leq 0, \quad x \in\left(0, x_{0}\right], \\
\Phi(x, v) \leq \Phi(x, 0), \quad x, v>0
\end{gathered}
$$

Therefore, if $R_{0} \leq 1$, we get $R_{0} \frac{\Phi(x, v)}{\Phi(x, 0)} \leq 1$ and then $\frac{d W_{0}}{d t} \leq 0$ for all $x, v, z>0$. By Theorem 5.3.1 in [17], the solutions of system (2.1)-(2.4) limit to $M$, the largest invariant subset of $\left\{\frac{d W_{0}}{d t}=0\right\}$. Clearly, it follows from (2.13) that $\frac{d W_{0}}{d t}=0$ if and only if $x=x_{0}, v=0$ and $z=0$. Noting that $M$ is invariant, for each element of $M$ 
we have $v=0$, and $z=0$, then $\dot{v}=0$. From Eq. (2.3) we drive that

$$
0=\dot{v}=N a \int_{0}^{\omega} g(\tau) e^{-n \tau} y(t-\tau) d \tau .
$$

This yields $y=0$. Hence $\frac{d W_{0}}{d t}=0$ if and only if $x=x_{0}, y=0, v=0$ and $z=0$. From LaSalle's invariance principle, $E_{0}$ is GAS.

Theorem 2. If Assumptions A1-A4 hold true and $R_{1} \leq 1<R_{0}$, then $E_{1}$ is GAS.

Proof. We construct the following Lyapunov functional

$$
\begin{aligned}
W_{1}= & N F G\left[x-x_{1}-\int_{x_{1}}^{x} \frac{\Phi\left(x_{1}, v_{1}\right)}{\Phi\left(\eta, v_{1}\right)} d \eta+\frac{1}{F} y_{1} H\left(\frac{y}{y_{1}}\right)\right. \\
& +\frac{\Phi\left(x_{1}, v_{1}\right) v_{1}}{F} \int_{0}^{h} f(\tau) e^{-m \tau} \int_{0}^{\tau} H\left(\frac{\Phi(x(t-\theta), v(t-\theta)) v(t-\theta)}{\Phi\left(x_{1}, v_{1}\right) v_{1}}\right) d \theta d \tau \\
& \left.+\frac{a y_{1}}{F G} \int_{0}^{\omega} g(\tau) e^{-n \tau} \int_{0}^{\tau} H\left(\frac{y(t-\theta)}{y_{1}}\right) d \theta d \tau\right]+v_{1} H\left(\frac{v}{v_{1}}\right)+\frac{q}{r} z
\end{aligned}
$$

The time derivative of $W_{1}$ along the trajectories of (2.1)-(2.4) is given by

$$
\begin{aligned}
\frac{d W_{1}}{d t}= & N F G\left[\left(1-\frac{\Phi\left(x_{1}, v_{1}\right)}{\Phi\left(x, v_{1}\right)}\right)(\lambda-d x-\Phi(x, v) v)\right. \\
& +\frac{1}{F}\left(1-\frac{y_{1}}{y}\right)\left(\int_{0}^{h} f(\tau) e^{-m \tau} \Phi(x(t-\tau), v(t-\tau)) v(t-\tau) d \tau-a y\right) \\
& +\frac{1}{F} \int_{0}^{h} f(\tau) e^{-m \tau}(\Phi(x, v) v-\Phi(x(t-\tau), v(t-\tau)) v(t-\tau) \\
& \left.+\Phi\left(x_{1}, v_{1}\right) v_{1} \ln \left(\frac{\Phi(x(t-\tau), v(t-\tau)) v(t-\tau)}{\Phi(x, v) v}\right)\right) d \tau \\
& \left.\left.+\frac{a}{F G} \int_{0}^{\omega} g(\tau) e^{-n \tau}\left(y-y(t-\tau)+y_{1} \ln \left(\frac{y(t-\tau)}{y}\right)\right) d \tau\right]\right] \\
& +\left(1-\frac{v_{1}}{v}\right)\left(N a \int_{0}^{\omega} g(\tau) e^{-n \tau} y(t-\tau) d \tau-c v-q v z\right)+q v z-\frac{q \mu}{r} z .
\end{aligned}
$$


Using the steady state conditions for $E_{1}$ :

$$
\lambda=d x_{1}+\frac{a}{F} y_{1}, \quad F \Phi\left(x_{1}, v_{1}\right) v_{1}=a y_{1}, \quad c v_{1}=N a G y_{1},
$$

we have

$$
\begin{aligned}
\frac{d W_{1}}{d t}= & N F G\left[d\left(x_{1}-x\right)\left(1-\frac{\Phi\left(x_{1}, v_{1}\right)}{\Phi\left(x, v_{1}\right)}\right)+\frac{a}{F} y_{1}\right. \\
& -\frac{a}{F} y_{1} \frac{\Phi\left(x_{1}, v_{1}\right)}{\Phi\left(x, v_{1}\right)}+\frac{a}{F} y_{1} \frac{\Phi(x, v) v}{\Phi\left(x, v_{1}\right) v_{1}} \\
& -\frac{a}{F^{2}} y_{1} \int_{0}^{h} f(\tau) e^{-m \tau} \frac{y_{1} \Phi(x(t-\tau), v(t-\tau)) v(t-\tau)}{y \Phi\left(x_{1}, v_{1}\right) v_{1}} d \tau+\frac{a}{F} y_{1} \\
& +\frac{a}{F^{2}} y_{1} \int_{0}^{h} f(\tau) e^{-m \tau} \ln \left(\frac{\Phi(x(t-\tau), v(t-\tau)) v(t-\tau)}{\Phi(x, v) v}\right) d \tau \\
& +\frac{a y_{1}}{F G} \int_{0}^{\omega} g(\tau) e^{-n \tau} \ln \left(\frac{y(t-\tau)}{y}\right) d \tau-\frac{a y_{1}}{F G} \int_{0}^{\omega} g(\tau) e^{-n \tau} \frac{v_{1} y(t-\tau)}{v y_{1}} d \tau \\
& \left.-\frac{a}{F} y_{1} \frac{v}{v_{1}}+\frac{a}{F} y_{1}\right]+q\left(v_{1}-\frac{\mu}{r}\right) z .
\end{aligned}
$$

Using the following equalities:

$$
\begin{aligned}
\ln \left(\frac{\Phi(x(t-\tau), v(t-\tau)) v(t-\tau)}{\Phi(x, v) v}\right)= & \ln \left(\frac{y_{1} \Phi(x(t-\tau), v(t-\tau)) v(t-\tau)}{y \Phi\left(x_{1}, v_{1}\right) v_{1}}\right) \\
& +\ln \left(\frac{\Phi\left(x_{1}, v_{1}\right)}{\Phi\left(x, v_{1}\right)}\right)+\ln \left(\frac{\Phi\left(x, v_{1}\right)}{\Phi(x, v)}\right)+\ln \left(\frac{v_{1} y}{v y_{1}}\right), \\
\ln \left(\frac{y(t-\tau)}{y}\right)= & \ln \left(\frac{v y_{1}}{v_{1} y}\right)+\ln \left(\frac{v_{1} y(t-\tau)}{v y_{1}}\right),
\end{aligned}
$$

we obtain

$$
\begin{aligned}
\frac{d W_{1}}{d t}= & N F G\left[d\left(x_{1}-x\right)\left(1-\frac{\Phi\left(x_{1}, v_{1}\right)}{\Phi\left(x, v_{1}\right)}\right)-\frac{a y_{1}}{F}\left(\frac{\Phi\left(x_{1}, v_{1}\right)}{\Phi\left(x, v_{1}\right)}-1-\ln \left(\frac{\Phi\left(x_{1}, v_{1}\right)}{\Phi\left(x, v_{1}\right)}\right)\right)\right. \\
& +\frac{a y_{1}}{F}\left(\frac{\Phi(x, v) v}{\Phi\left(x, v_{1}\right) v_{1}}-\frac{v}{v_{1}}+\frac{\Phi\left(x, v_{1}\right)}{\Phi(x, v)}-1\right) \\
& -\frac{a y_{1}}{F}\left(\frac{\Phi\left(x, v_{1}\right)}{\Phi(x, v)}-1-\ln \left(\frac{\Phi\left(x, v_{1}\right)}{\Phi(x, v)}\right)\right) \\
& -\frac{a y_{1}}{F^{2}} \int_{0}^{h} f(\tau) e^{-m \tau}\left(\frac{y_{1} \Phi(x(t-\tau), v(t-\tau)) v(t-\tau)}{y\left(x_{1}, v_{1}\right) v_{1}}-1\right.
\end{aligned}
$$




$$
\begin{aligned}
& \left.-\ln \left(\frac{y_{1} \Phi(x(t-\tau), v(t-\tau)) v(t-\tau)}{y \Phi\left(x_{1}, v_{1}\right) v_{1}}\right)\right) d \tau \\
& \left.-\frac{a y_{1}}{F G} \int_{0}^{\omega} g(\tau) e^{-n \tau}\left(\frac{v_{1} y(t-\tau)}{v y_{1}}-1-\ln \left(\frac{v_{1} y(t-\tau)}{v y_{1}}\right)\right) d \tau\right]+q\left(v_{1}-\frac{\mu}{r}\right) z .
\end{aligned}
$$

Eq. (2.17) can be rewritten as:

$$
\begin{aligned}
\frac{d W_{1}}{d t}= & N F G\left[\frac{d}{\Phi\left(x, v_{1}\right)}\left(x_{1}-x\right)\left(\Phi\left(x, v_{1}\right)-\Phi\left(x_{1}, v_{1}\right)\right)\right. \\
& +\frac{\Phi\left(x_{1}, v_{1}\right)}{\Phi(x, v) \Phi\left(x, v_{1}\right)}\left(\Phi(x, v)-\Phi\left(x, v_{1}\right)\right)\left(\Phi(x, v) v-\Phi\left(x, v_{1}\right) v_{1}\right) \\
& -\frac{a y_{1}}{F} H\left(\frac{\Phi\left(x_{1}, v_{1}\right)}{\Phi\left(x, v_{1}\right)}\right)-\frac{a y_{1}}{F} H\left(\frac{\Phi\left(x, v_{1}\right)}{\Phi(x, v)}\right) \\
& -\frac{a y_{1}}{F^{2}} \int_{0}^{h} f(\tau) e^{-m \tau} H\left(\frac{y_{1} \Phi(x(t-\tau), v(t-\tau)) v(t-\tau)}{y \Phi\left(x_{1}, v_{1}\right) v_{1}}\right) d \tau \\
& \left.-\frac{a y_{1}}{F G} \int_{0}^{\omega} g(\tau) e^{-n \tau} H\left(\frac{v_{1} y(t-\tau)}{v y_{1}}\right) d \tau\right] \\
& +q z\left(v_{1}-\frac{\mu}{r}\right) .
\end{aligned}
$$

From Assumptions A3-A4, we have

$$
\begin{array}{r}
\left(x_{1}-x\right)\left(\Phi\left(x, v_{1}\right)-\Phi\left(x_{1}, v_{1}\right)\right) \leq 0 \\
\left(\Phi(x, v)-\Phi\left(x, v_{1}\right)\right)\left(\Phi(x, v) v-\Phi\left(x, v_{1}\right) v_{1}\right) \leq 0 .
\end{array}
$$

Now we show that if $R_{1} \leq 1$ then $v_{1} \leq \frac{\mu}{r}=v_{2}$. Let $R_{0}>1$, then we want to show that

$$
\operatorname{sgn}\left(x_{2}-x_{1}\right)=\operatorname{sgn}\left(v_{1}-v_{2}\right)=\operatorname{sgn}\left(R_{1}-1\right) .
$$

From Assumptions A2-A4, for $x_{1}, x_{2}, v_{1}, v_{2}>0$, we have:

$$
\begin{gathered}
\left(\Phi\left(x_{2}, v_{1}\right)-\Phi\left(x_{1}, v_{1}\right)\right)\left(x_{2}-x_{1}\right)>0, \\
\left(\Phi\left(x_{2}, v_{2}\right) v_{2}-\Phi\left(x_{2}, v_{1}\right) v_{1}\right)\left(v_{2}-v_{1}\right)>0 \\
\left(\Phi\left(x_{2}, v_{2}\right)-\Phi\left(x_{2}, v_{1}\right)\right)\left(v_{1}-v_{2}\right)>0 .
\end{gathered}
$$

Suppose that, $\operatorname{sgn}\left(x_{2}-x_{1}\right)=\operatorname{sgn}\left(v_{2}-v_{1}\right)$. Using the conditions of the steady states $E_{1}$ and $E_{2}$ we have

$$
\begin{aligned}
\left(\lambda-d x_{2}\right)-\left(\lambda-d x_{1}\right) & =\Phi\left(x_{2}, v_{2}\right) v_{2}-\Phi\left(x_{1}, v_{1}\right) v_{1} \\
& =\Phi\left(x_{2}, v_{2}\right) v_{2}-\Phi\left(x_{2}, v_{1}\right) v_{1}+\left[\Phi\left(x_{2}, v_{1}\right)-\Phi\left(x_{1}, v_{1}\right)\right] v_{1}
\end{aligned}
$$


but

$$
\left[\left(\lambda-d x_{2}\right)-\left(\lambda-d x_{1}\right)\right]\left(x_{1}-x_{2}\right)=d\left(x_{2}-x_{1}\right)^{2}>0 .
$$

Then from (2.19) and (2.20) we get:

$$
\operatorname{sgn}\left(x_{1}-x_{2}\right)=\operatorname{sgn}\left(x_{2}-x_{1}\right),
$$

which leads to contradiction. Thus, $\operatorname{sgn}\left(x_{2}-x_{1}\right)=\operatorname{sgn}\left(v_{1}-v_{2}\right)$. Using the steady state conditions for $E_{1}$ we have $\frac{N F G \Phi\left(x_{1}, v_{1}\right)}{c}=1$, then

$$
\begin{aligned}
R_{1}-1 & =\frac{N F G}{c}\left(\Phi\left(x_{2}, v_{2}\right)-\Phi\left(x_{1}, v_{1}\right)\right) \\
& =\frac{N F G}{c}\left(\Phi\left(x_{2}, v_{2}\right)-\Phi\left(x_{2}, v_{1}\right)+\Phi\left(x_{2}, v_{1}\right)-\Phi\left(x_{1}, v_{1}\right)\right) \\
& =\frac{N F G}{c}\left[\Phi\left(x_{2}, v_{2}\right)-\Phi\left(x_{2}, v_{1}\right)+\Phi\left(x_{2}, v_{1}\right)-\Phi\left(x_{1}, v_{1}\right)\right] .
\end{aligned}
$$

From (2.19) and (2.21), we get

$$
\operatorname{sgn}\left(R_{1}-1\right)=\operatorname{sgn}\left(v_{1}-v_{2}\right) .
$$

Hence, if $R_{0}>1$, then $x_{1}, y_{1}, v_{1}>0$, and if $R_{1} \leq 1$, then $v_{1} \leq v_{2}=\frac{\mu}{r}$ and $\frac{d W_{1}}{d t} \leq 0$ for all $x, y, v, z>0$. By Theorem 5.3.1 in [17], the solutions of system (2.1)-(2.4) limit to $M$, the largest invariant subset of $\left\{\frac{d W_{1}}{d t}=0\right\}$. It can be seen that $\frac{d W_{1}}{d t}=0$ if and only if $x=x_{1}, v=v_{1}, z=0$ and $H=0$ i.e.

$$
\frac{y_{1} \Phi(x(t-\tau), v(t-\tau)) v(t-\tau)}{y \Phi\left(x_{1}, v_{1}\right) v_{1}}=\frac{v_{1} y(t-\tau)}{v y_{1}}=1 \text { for almost all } \tau \in[0, \rho] .
$$

From Eq. (2.23), if $v=v_{1}$ then $y=y_{1}$ and hence $\frac{d W_{1}}{d t}$ equal to zero at $E_{1}$. LaSalle's invariance principle implies global stability of $E_{1}$.

Theorem 3. If Assumptions Al-A4 hold true and $R_{1}>1$, then $E_{2}$ is GAS.

Proof. We construct the following Lyapunov functional

$$
\begin{aligned}
W_{2}= & N F G\left[x-x_{2}-\int_{x_{2}}^{x} \frac{\Phi\left(x_{2}, v_{2}\right)}{\Phi\left(\eta, v_{2}\right)} d \eta+\frac{1}{F} y_{2} H\left(\frac{y}{y_{2}}\right)\right. \\
& +\frac{\Phi\left(x_{2}, v_{2}\right) v_{2}}{F} \int_{0}^{h} f(\tau) e^{-m \tau} \int_{0}^{\tau} H\left(\frac{\Phi(x(t-\theta), v(t-\theta)) v(t-\theta)}{\Phi\left(x_{2}, v_{2}\right) v_{2}}\right) d \theta d \tau \\
& \left.+\frac{a y_{2}}{F G} \int_{0}^{\omega} g(\tau) e^{-n \tau} \int_{0}^{\tau} H\left(\frac{y(t-\theta)}{y_{2}}\right) d \theta d \tau\right]+v_{2} H\left(\frac{v}{v_{2}}\right)+\frac{q}{r} z_{2} H\left(\frac{z}{z_{2}}\right) .
\end{aligned}
$$


Calculating the time derivative of $W_{2}$ along the solution of (2.1)-(2.4) we obtain

$$
\begin{aligned}
\frac{d W_{2}}{d t}= & N F G\left[\left(1-\frac{\Phi\left(x_{2}, v_{2}\right)}{\Phi\left(x, v_{2}\right)}\right)(\lambda-d x-\Phi(x, v) v)\right. \\
& +\frac{1}{F}\left(1-\frac{y_{2}}{y}\right)\left(\int_{0}^{h} f(\tau) e^{-m \tau} \Phi(x(t-\tau), v(t-\tau)) v(t-\tau) d \tau-a y\right) \\
& +\frac{1}{F} \int_{0}^{h} f(\tau) e^{-m \tau}(\Phi(x, v) v-\Phi(x(t-\tau), v(t-\tau)) v(t-\tau) \\
& \left.+\Phi\left(x_{2}, v_{2}\right) v_{2} \ln \left(\frac{\Phi(x(t-\tau), v(t-\tau)) v(t-\tau)}{\Phi(x, v) v}\right)\right) d \tau \\
& \left.+\frac{a}{F G} \int_{0}^{\omega} g(\tau) e^{-n \tau}\left(y-y(t-\tau)+y_{2} \ln \left(\frac{y(t-\tau)}{y}\right)\right) d \tau\right] \\
& +\left(1-\frac{v_{2}}{v}\right)\left(N a \int_{0}^{\omega} g(\tau) e^{-n \tau} y(t-\tau) d \tau-c v-q v z\right) \\
& +\left(1-\frac{z_{2}}{z}\right)\left(q v z-\frac{q \mu}{r} z\right) .
\end{aligned}
$$

Applying $\lambda=d x_{2}+\Phi\left(x_{2}, v_{2}\right) v_{2}$ we obtain

$$
\begin{aligned}
\frac{d W_{2}}{d t}= & N F G\left[d\left(x_{2}-x\right)\left(1-\frac{\Phi\left(x_{2}, v_{2}\right)}{\Phi\left(x, v_{2}\right)}\right)+\Phi\left(x_{2}, v_{2}\right) v_{2}-\Phi\left(x_{2}, v_{2}\right) v_{2} \frac{\Phi\left(x_{2}, v_{2}\right)}{\Phi\left(x, v_{2}\right)}\right. \\
& +\Phi(x, v) v \frac{\Phi\left(x_{2}, v_{2}\right)}{\Phi\left(x, v_{2}\right)} \\
& -\frac{1}{F} \Phi\left(x_{2}, v_{2}\right) v_{2} \int_{0}^{h} f(\tau) e^{-m \tau} \frac{y_{2} \Phi(x(t-\tau), v(t-\tau)) v(t-\tau)}{y\left(x_{2}, v_{2}\right) v_{2}} d \tau+\frac{a}{F} y_{2} \\
& +\frac{1}{F} \Phi\left(x_{2}, v_{2}\right) v_{2} \int_{0}^{h} f(\tau) e^{-m \tau} \ln \left(\frac{\Phi(x(t-\tau), v(t-\tau)) v(t-\tau)}{\Phi(x, v) v}\right) d \tau \\
& \left.+\frac{a y_{2}}{F G} \int_{0}^{\omega} g(\tau) e^{-n \tau} \ln \left(\frac{y(t-\tau)}{y}\right) d \tau-\frac{a y_{2}}{F G} \int_{0}^{\omega} g(\tau) e^{-n \tau} \frac{v_{2} y(t-\tau)}{v y_{2}} d \tau\right] \\
& -c v+c v_{2}+q v_{2} z-q v z_{2}-\frac{q \mu}{r} z+\frac{q \mu}{r} z_{2} .
\end{aligned}
$$


Using the steady state conditions of $E_{2}$

$$
F \Phi\left(x_{2}, v_{2}\right) v_{2}=a y_{2}, \quad c v_{2}=N a G y_{2}-q v_{2} z_{2}, \quad \mu=r v_{2},
$$

and the following equalities:

$$
\begin{aligned}
c v & =c v_{2} \frac{v}{v_{2}}=N F G\left(\frac{a}{F} y_{2} \frac{v}{v_{2}}\right)-q v z_{2}, \\
\ln \left(\frac{\Phi(x(t-\tau), v(t-\tau)) v(t-\tau)}{\Phi(x, v) v}\right)= & \ln \left(\frac{y_{2} \Phi(x(t-\tau), v(t-\tau)) v(t-\tau)}{y \Phi\left(x_{2}, v_{2}\right) v_{2}}\right) \\
& +\ln \left(\frac{\Phi\left(x_{2}, v_{2}\right)}{\Phi\left(x, v_{2}\right)}\right)+\ln \left(\frac{\Phi\left(x, v_{2}\right)}{\Phi(x, v)}\right)+\ln \left(\frac{v_{2} y}{v y_{2}}\right), \\
\ln \left(\frac{y(t-\tau)}{y}\right)= & \ln \left(\frac{v y_{2}}{v_{2} y}\right)+\ln \left(\frac{v_{2} y(t-\tau)}{v y_{2}}\right),
\end{aligned}
$$

we obtain

$$
\begin{aligned}
\frac{d W_{2}}{d t}= & N F G\left[d\left(x_{2}-x\right)\left(1-\frac{\Phi\left(x_{2}, v_{2}\right)}{\Phi\left(x, v_{2}\right)}\right)-\frac{a y_{2}}{F}\left(\frac{\Phi\left(x_{2}, v_{2}\right)}{\Phi\left(x, v_{2}\right)}-1-\ln \left(\frac{\Phi\left(x_{2}, v_{2}\right)}{\Phi\left(x, v_{2}\right)}\right)\right)\right. \\
& -\frac{a y_{2}}{F^{2}} \int_{0}^{h} f(\tau) e^{-m \tau}\left(\frac{y_{2} \Phi(x(t-\tau), v(t-\tau)) v(t-\tau)}{y \Phi\left(x_{2}, v_{2}\right) v_{2}}-1\right. \\
& \left.-\ln \left(\frac{y_{2} \Phi(x(t-\tau), v(t-\tau)) v(t-\tau)}{y \Phi\left(x_{2}, v_{2}\right) v_{2}}\right)\right) d \tau \\
& -\frac{a y_{2}}{F G} \int_{0}^{\omega} g(\tau) e^{-n \tau}\left(\frac{v_{2} y(t-\tau)}{v y_{2}}-1-\ln \left(\frac{v_{2} y(t-\tau)}{v y_{2}}\right)\right) d \tau \\
& -\frac{a y_{2}}{F}\left(\frac{\Phi\left(x, v_{2}\right)}{\Phi(x, v)}-1-\ln \left(\frac{\Phi\left(x, v_{2}\right)}{\Phi(x, v)}\right)\right) \\
& \left.+\frac{a y_{2}}{F}\left(\frac{\Phi(x, v) v}{\Phi\left(x, v_{2}\right) v_{2}}+\frac{\Phi\left(x, v_{2}\right)}{\Phi(x, v)}-\frac{v}{v_{2}}-1\right)\right] .
\end{aligned}
$$

We can rewrite (2.27) as

$$
\begin{aligned}
\frac{d W_{2}}{d t}= & N F G\left[\frac{d}{\Phi\left(x, v_{2}\right)}\left(x_{2}-x\right)\left(\Phi\left(x, v_{2}\right)-\Phi\left(x_{2}, v_{2}\right)\right)-\frac{a y_{2}}{F} H\left(\frac{\Phi\left(x_{2}, v_{2}\right)}{\Phi\left(x, v_{2}\right)}\right)\right. \\
& -\frac{a y_{2}}{F^{2}} \int_{0}^{h} f(\tau) e^{-m \tau} H\left(\frac{y_{2} \Phi(x(t-\tau), v(t-\tau)) v(t-\tau)}{y \Phi\left(x_{2}, v_{2}\right) v_{2}}\right) d \tau \\
& -\frac{a y_{2}}{F G} \int_{0}^{\omega} g(\tau) e^{-n \tau} H\left(\frac{v_{2} y(t-\tau)}{v y_{2}}\right) d \tau-\frac{a y_{2}}{F} H\left(\frac{\Phi\left(x, v_{2}\right)}{\Phi(x, v)}\right) \\
& \left.+\frac{\Phi\left(x_{2}, v_{2}\right)}{\Phi(x, v) \Phi\left(x, v_{2}\right)}\left(\Phi(x, v)-\Phi\left(x, v_{2}\right)\right)\left(\Phi(x, v) v-\Phi\left(x, v_{2}\right) v_{2}\right)\right] .
\end{aligned}
$$


From Assumptions A3-A4, we have

$$
\begin{aligned}
\left(x_{2}-x\right)\left(\Phi\left(x, v_{2}\right)-\Phi\left(x_{2}, v_{2}\right)\right) & \leq 0 \\
\left(\Phi(x, v)-\Phi\left(x, v_{2}\right)\right)\left(\Phi(x, v) v-\Phi\left(x, v_{2}\right) v_{2}\right) & \leq 0 .
\end{aligned}
$$

Therefore, $\frac{d W_{2}}{d t} \leq 0$. One can easily show that $\frac{d W_{2}}{d t}=0$ occurs at $E_{2}$. LaSalle's invariance principle implies global stability of $E_{2}$.

\section{NUMERICAL RESULTS AND DISCUSSIONS}

In this section we first show two special forms of the general function $\Phi(x, v)$ which satisfy Assumptions A1-A4, then perform some numerical simulations for model (2.1)-(2.4) in case of discrete-time delays. Function $\Phi(x, v)$ can be chosen as:

(1) Beddington-DeAngelis functional response:

$$
\Phi_{1}(x, v)=\frac{\beta x}{1+\gamma x+\alpha v},
$$

where $\alpha, \gamma \geq 0$. We have the following

$$
\begin{aligned}
\Phi_{1}(x, v) & >0 \text { for all } x>0, v>0, \text { and } \Phi_{1}(0, v)=0, \\
\frac{\partial \Phi_{1}(x, v)}{\partial x} & =\frac{\beta(1+\alpha v)}{(1+\gamma x+\alpha v)^{2}}>0 \text { for all } v>0, \\
\frac{\partial \Phi_{1}(x, v)}{\partial v} & =\frac{-\beta \alpha x}{(1+\gamma x+\alpha v)^{2}}<0 \text { for all } x>0, \\
\frac{\partial\left(\Phi_{1}(x, v) v\right)}{\partial v} & =\frac{\beta x(1+\gamma x)}{(1+\gamma x+\alpha v)^{2}}>0 \text { for all } x>0 .
\end{aligned}
$$

Then function $\Phi(x, v)$ satisfies Assumptions A1-A4 and the global stability results presented in Theorems 1-3 are valid.

(2) Crowley-Martin functional response:

$$
\Phi_{2}(x, v)=\frac{\beta x}{(1+\gamma x)(1+\alpha v)} .
$$

Function $\Phi_{2}$ satisfies the following:

$$
\begin{aligned}
\Phi_{2}(x, v) & >0 \text { for all } x>0, v>0, \text { and } \Phi_{2}(0, v)=0, \\
\frac{\partial \Phi_{2}(x, v)}{\partial x} & =\frac{\beta}{(1+\alpha v)(1+\gamma x)^{2}}>0 \text { for all } v>0, \\
\frac{\partial \Phi_{2}(x, v)}{\partial v} & =\frac{-\beta \alpha x}{(1+\alpha v)^{2}(1+\gamma x)}<0 \text { for all } x>0, \\
\frac{\partial\left(\Phi_{2}(x, v) v\right)}{\partial v} & =\frac{\beta x}{(1+\alpha v)^{2}(1+\gamma x)}>0 \text { for all } x>0 .
\end{aligned}
$$

Thus Assumption A1-A4 hold true and Theorems 1-3 are applicable. 
Next, we shall perform simulation studies for the model (2.1)-(2.4) with function $\Phi_{2}(x, v)$ and with particular distribution functions $f(\tau)$ and $g(\tau)$. All computations are carried out by MATLAB. We are going to choose the probability distribution functions $f(\tau)$ and $g(\tau)$ as $f(\tau)=\delta\left(\tau-\tau_{1}\right)$ and $g(\tau)=\delta\left(\tau-\tau_{2}\right)$, where $\delta($.$) is the$ dirac delta function, $\tau_{1}$ and $\tau_{2}$ are constants and $\tau_{1} \in[0, h], \tau_{2} \in[0, \omega]$. We can see that, from the properties of dirac delta function, as $h$ and $\omega$ tend to $\infty$,

$$
\begin{aligned}
\int_{0}^{\infty} f(\tau) d \tau & =\int_{0}^{\infty} g(\tau) d \tau=1 \\
F & =\int_{0}^{\infty} \delta\left(\tau-\tau_{1}\right) e^{-m \tau} d \tau=e^{-m \tau_{1}}, \\
G & =\int_{0}^{\infty} \delta\left(\tau-\tau_{2}\right) e^{-n \tau} d \tau=e^{-n \tau_{2}} .
\end{aligned}
$$

Moreover, we have

$$
\begin{aligned}
& \begin{aligned}
\int_{0}^{\infty} \delta\left(\tau-\tau_{1}\right) e^{-m \tau} \Phi_{2}(x(t-\tau), v(t-\tau)) v(t-\tau) d \tau & \\
& =e^{-m \tau_{1}} \Phi_{2}\left(x\left(t-\tau_{1}\right), v\left(t-\tau_{1}\right)\right) v\left(t-\tau_{1}\right), \\
\int_{0}^{\infty} \delta\left(\tau-\tau_{2}\right) e^{-n \tau} y(t-\tau) d \tau & =e^{-n \tau_{2}} y\left(t-\tau_{2}\right) .
\end{aligned}
\end{aligned}
$$

Using these choices, model (2.1)-(2.4) becomes

$$
\begin{aligned}
\dot{x}(t) & =\lambda-d x(t)-\frac{\beta x(t) v(t)}{(1+\gamma x(t))(1+\alpha v(t))}, \\
\dot{y}(t) & =e^{-m \tau_{1}} \frac{\beta x\left(t-\tau_{1}\right) v\left(t-\tau_{1}\right)}{\left(1+\gamma x\left(t-\tau_{1}\right)\right)\left(1+\alpha v\left(t-\tau_{1}\right)\right)}-a y(t), \\
\dot{v}(t) & =N a e^{-n \tau_{2}} y\left(t-\tau_{2}\right)-c v(t)-q v(t) z(t), \\
\dot{z}(t) & =r v(t) z(t)-\mu z(t) .
\end{aligned}
$$

As a result, the parameters $R_{0}$ and $R_{1}$ become

$$
\begin{gathered}
R_{0}=\frac{e^{-\left(m \tau_{1}+n \tau_{2}\right)} N \beta x_{0}}{c\left(1+\gamma x_{0}\right)}, \\
R_{1}=\frac{e^{-\left(m \tau_{1}+n \tau_{2}\right)} N \beta x_{2}}{c\left(1+\gamma x_{2}\right)\left(1+\alpha v_{2}\right)},
\end{gathered}
$$


where

$$
\begin{aligned}
x_{2}= & \frac{1}{2 \gamma\left(1+\alpha v_{2}\right)}\left(\gamma x_{0}\left(1+\alpha v_{2}\right)-\left(1+\zeta v_{2}\right)\right. \\
& \left.+\sqrt{\left[\left(1+\zeta v_{2}\right)-\gamma x_{0}\left(1+\zeta v_{2}\right)\right]^{2}+4 \gamma x_{0}\left(1+\alpha v_{2}\right)^{2}}\right), \\
v_{2}= & \mu / r
\end{aligned}
$$

and $\zeta=\alpha+\frac{\beta}{d}$.

Now we will perform some numerical simulations to testify our theoretical results. The values of the parameters of model (3.1)-(3.4) are given in Table 1.

TABLE 1. The values of the parameters of model (3.1)-(3.4).

\begin{tabular}{||c|c||c|c||c|c||}
\hline Parameter & Value & Parameter & Value & Parameter & value \\
\hline$\lambda$ & 10 & $a$ & 0.1 & $\mu$ & 0.1 \\
\hline$d$ & 0.01 & $N$ & 10 & $m$ & 1 \\
\hline$\beta$ & Varied & $c$ & 3 & $n$ & 3.4 \\
\hline$\gamma$ & 0.0001 & $q$ & 0.01 & $\tau_{1}$ & 1 \\
\hline$\alpha$ & 0.0001 & $r$ & Varied & $\tau_{2}$ & 1.2 \\
\hline
\end{tabular}

Now, we study the following cases:

- $R_{0} \leq 1$. We choose, $\beta=0.001$ and $r=0.01$. Using these data, we compute $R_{0}=0.0188$ and $R_{1}=0.0101$. According to Theorem $1, E_{0}$ is GAS. Figures 1-4 show that, the numerical results are consistent with Theorem 1 . We can see that, the concentration of uninfected cells is increased and converged to its normal value $\frac{\lambda}{d}=1000$, while the concentrations of infected cells, free viruses and B cells are decaying and approaching zero. In this case the viruses can be cleared from the body.

- $R_{1} \leq 1<R_{0}$. We take $\beta=0.1$ and $r=0.01$. In this case, $R_{0}=1.8848$ and $R_{1}=0.0205$. From Theorem 2, $E_{1}$ is GAS. Figures 1-4 show that the numerical results are consistent with Theorem 2. We can see that, the trajectory of the system will tend to the infected steady state without humoral immune response $E_{1}(506.77,18.1449,0.1023,0)$, and the infection becomes chronic but with no persistent humoral immune response.

- $R_{1}>1$. We choose, $\beta=0.1$ and $r=1$. Then, we compute $R_{0}=1.8848$ and $R_{1}=1.0107$. From Theorem 3, $E_{2}$ is GAS. Figures 1-4 demonstrate that, our simulations are consistent with the theoretical results of Theorem 3 . We observe that, the trajectory of the system will tend to the infected steady state with humoral immune response $E_{2}(512.4948,17.9343,0.1,3.2238)$. Then, the infection becomes chronic but with persistent humoral immune response. From Figures 1 and 3 we observe that, if $R_{1}>1$ the humoral immune response reduce the concentration of free viruses and increase the concentration of uninfected cells. 


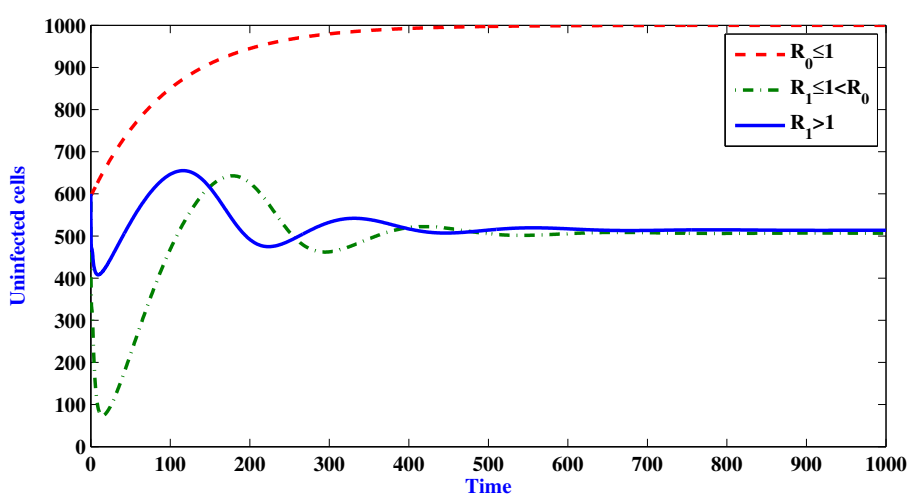

FIGURE 1. Evaluation of uninfected cells.

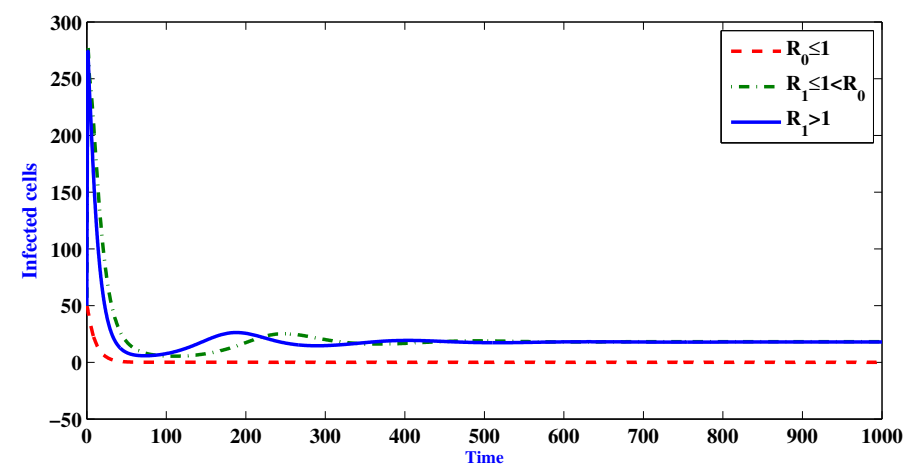

FIGURE 2. Evaluation of infected cells.

We note that, the values of the parameters $q, r$ and $\mu$ have no impact on the value of $R_{0}$, since $R_{0}$ is independent of those parameters. This fact seems to suggest that, humoral immune response do not play a role in eliminating the viruses. From above we can see that, $R_{1}$ can be increased by increasing the parameter $r$. When we compare the cases $R_{1} \leq 1<R_{0}$ and $R_{1}>1$, we can see that, the presence of humoral immune response (i.e. $R_{1}>1$ ) reduce the concentration of free viruses and infected cells and increase the concentration of uninfected cells. It means that, the humoral immune response can play an important role in controlling the infection.

\section{CONCLUSION}

In this paper, we have proposed a virus infection model describing the interaction of the virus with target cell taking into account the humoral immune response. The 


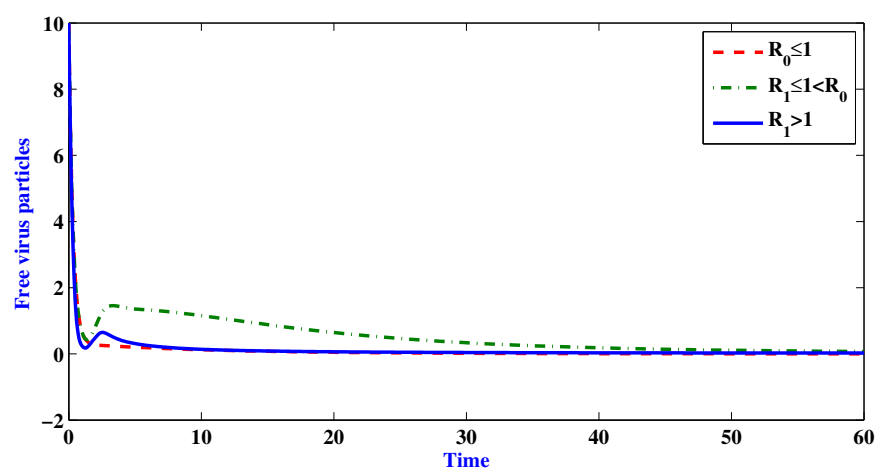

FIGURE 3. Evaluation of free virus particles.

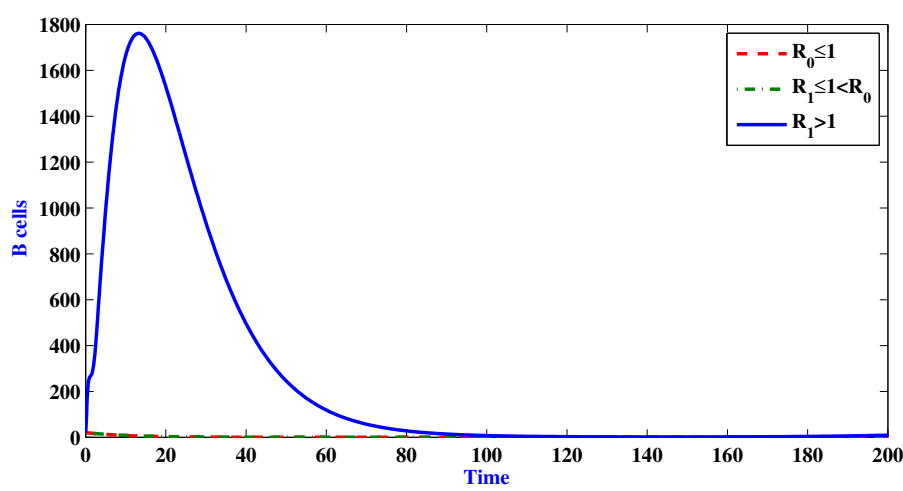

FIGURE 4. Evaluation of B cells.

infection rate is given by a general function. Two types of distributed time delays describing the time needed for infection of target cell and virus replication have been incorporated into the model. Using the method of Lyapunov functional, we have established that the global dynamics of the model is determined by two threshold parameters $R_{0}$ and $R_{1}$. We have proven that if $R_{0} \leq 1$, then the uninfected steady state $E_{0}$ is GAS, and the viruses are cleared, if $R_{1} \leq 1<R_{0}$, then the infected steady state without humoral immune response $E_{1}$ is GAS, and the infection becomes chronic but with no persistent $\mathrm{B}$ cells response, and if $R_{1}>1$, then the infected steady state with B cells response $E_{2}$ is GAS, and the infection is chronic with persistent B cells response. Numerical simulations have been performed for the virus dynamics model with discrete-time delays and special form of the function $\Phi(x, v)$. Our simulation results confirm the theoretical results given in Theorems 1-3. 


\section{ACKNOWLEDGEMENTS}

The authors are grateful to the anonymous reviewers for constructive suggestions and valuable comments, which improve the quality of the article.

\section{REFERENCES}

[1] A. Alhejelan and A. M. Elaiw, "Global dynamics of virus infection model with humoral immune response and distributed delays," Journal of Computational Analysis and Applications, vol. 17, no. 3, pp. 515-523, 2014.

[2] R. M. Anderson, R. M. May, and S. Gupta, "Non-linear phenomena in host-parasite interactions," Parasitology, vol. 99, pp. 59-79, 1989, doi: 10.1017/S0031182000083426.

[3] D. S. Callaway and A. S. Perelson, "HIV-1 infection and low steady state viral loads," Bulletin of Mathematical Biology, vol. 64, pp. 29-64, 2002.

[4] R. V. Culshaw and S. Ruan, "A delay-differential equation model of HIV infection of CD4+ Tcells," Mathematical Biosciences, vol. 165, pp. 27-39, 2000, doi: 10.1016/S0025-5564(00)000067.

[5] J. A. Deans and S. Cohen, "Immunology of malaria," Annual Reviews in Microbiology, vol. 37, pp. $25-49,1983$.

[6] N. M. Dixit and A. S. Perelson, "Complex patterns of viral load decay under antiretroviral therapy: Influence of pharmacokinetics and intracellular delay," Journal of Theoretical Biology, vol. 226, pp. 95-109, 2004, doi: 10.1016/j.jtbi.2003.09.002.

[7] A. M. Elaiw, "Receding horizon control method applied to antiviral treatment of AIDS," Miskolc Mathematical Notes, vol. 5, no. 2, pp. 173-186, 2004.

[8] A. M. Elaiw, "Global properties of a class of HIV models," Nonlinear Analysis: Real World Applications, vol. 11, pp. 2253-2263, 2010, doi: 10.1016/j.nonrwa.2009.07.001.

[9] A. M. Elaiw, "Global dynamics of an HIV infection model with two classes of target cells and distributed delays," Discrete Dynamics in Nature and Society, vol. 2012, no. Article ID 253703, 13 pages, pp. 2425-2432, 2012, doi: 10.1155/2012/253703.

[10] A. M. Elaiw, "Global properties of a class of virus infection models with multitarget cells," Nonlinear Dynamics, vol. 69, no. 1, pp. 423-435, 2012, doi: 10.1007/s11071-011-0275-0.

[11] A. M. Elaiw and M. A. Alghamdi, "Global properties of virus dynamics models with multitarget cells and discrete-time delays," Discrete Dynamics in Nature and Society, vol. 69, pp. Article ID 201 274,19 pages, 2011, doi: 10.1155/2011/201274.

[12] A. M. Elaiw, A. Alhejelan, and M. A. Alghamdi, "Global dynamics of virus infection model with antibody immune response and distributed delays," Discrete Dynamics in Nature and Society, vol. 36, p. Article ID 781407, 2013, doi: 10.1155/2013/781407.

[13] A. M. Elaiw and N. A. Almuallem, "Global dynamics of delay-distributed HIV infection models with differential drug efficacy in cocirculating target cells," Mathematical Methods in the Applied Sciences, vol. 39, no. 1, pp. 4-31, 2016, doi: 10.1002/mma.3453.

[14] A. M. Elaiw and N. H. AlShamrani, "Global stability of humoral immunity virus dynamics models with nonlinear infection rate and removal," Nonlinear Analysis: Real World Applications, vol. 26, pp. 161-190, 2015, doi: 10.1016/j.nonrwa.2015.05.007.

[15] A. M. Elaiw, I. A. Hassanien, and S. A. Azoz, "Global stability of HIV infection models with intracellular delays," Journal of the Korean Mathematical Society, vol. 49, no. 4, pp. 779-794, 2012, doi: 10.4134/JKMS.2012.49.4.779.

[16] E. Gyurkovics and A. M. Elaiw, "A stabilizing sampled-data $\ell$-step receding horizon control with application to a HIV/AIDS model," Differential Equations and Dynamical Systems, vol. 14, pp. 1-30, 2006. 
[17] J. K. Hale, , and S. V. Lunel, Introduction to functional differential equations. Springer-Verlag, New York, 1993.

[18] G. Huang, Y. Takeuchi, and W. Ma, "Lyapunov functionals for delay differential equations model of viral infection," SIAM Journal on Applied Mathematics, vol. 70, no. 7, pp. 2693-2708, 2010, doi: $10.1137 / 090780821$.

[19] H. F. Huo, Y. L. Tang, and L. X. Feng, "A virus dynamics model with saturation infection and humoral immunity," Int. Journal of Math. Analysis, vol. 6, no. 40, pp. 1977-1983, 2012.

[20] A. Korobeinikov, "Global properties of infectious disease models with nonlinear incdence," Bulletin of Mathematical Biology, vol. 69, pp. 1871-1886, 2007, doi: 10.1007/s11538-007-9196-y.

[21] J. Li, K. Wang, and Y. Yang, "Dynamical behaviors of an HBV infection model with logistic hepatocyte growth," Mathematical and Computer Modelling, vol. 54, pp. 704-711, 2011, doi: 10.1016/j.mcm.2011.03.013.

[22] M. Y. Li and H. Shu, "Global dynamics of a mathematical model for HTLV-I infection of CD4+ T cells with delayed CTL response," Nonlinear Analysis: Real World Applications, vol. 13, pp. 1080-1092, 2012, doi: 10.1016/j.nonrwa.2011.02.026.

[23] J. Mittler, B. Sulzer, A. Neumann, and A. Perelson, "Influence of delayed virus production on viral dynamics in HIV-1 infected patients," Mathematical Biosciences, vol. 152, pp. 143-163, 1998, doi: 10.1016/S0025-5564(98)10027-5.

[24] A. Murase, T. Sasaki, and T. Kajiwara, "Stability analysis of pathogen-immune interaction dynamics," Journal of Mathematical Biology, vol. 51, pp. 247-267, 2005, doi: 10.1007/s00285-0050321-y.

[25] P. W. Nelson, J. Murray, and A. Perelson, "A model of HIV-1 pathogenesis that includes an intracellular delay," Mathematical Biosciences, vol. 163, pp. 201-215, 2000, doi: 10.1016/S00255564(99)00055-3.

[26] P. W. Nelson and A. S. Perelson, "Mathematical analysis of delay differential equation models of HIV-1 infection," Mathematical Biosciences, vol. 179, pp. 73-94, 2002, doi: 10.1016/S00255564(02)00099-8.

[27] A. U. Neumann, N. P. Lam, H. Dahari, D. R. Gretch, T. E. Wiley, T. J. Layden, and A. S. Perelson, "Hepatitis $\mathrm{C}$ viral dynamics in vivo and the antiviral efficacy of interferon-alpha therapy," Science, vol. 282, pp. 103-107, 1998, doi: 10.1126/science.282.5386.103.

[28] M. A. Nowak and C. R. M. Bangham, "Population dynamics of immune responses to persistent viruses," Science, vol. 272, no. 5258, pp. 74-79, 1996, doi: 10.1126/science.272.5258.74.

[29] M. A. Nowak and R. M. May, Virus dynamics: Mathematical Principles of Immunology and Virology. Oxford University Press, 2000.

[30] A. S. Perelson, "Modelling viral and immune system dynamics," Nature Reviews Immunology, vol. 2, pp. 28-36, 2002, doi: 10.1038/nri700.

[31] A. S. Perelson and P. W. Nelson, "Mathematical analysis of HIV-1 dynamics in vivo," Society for Industrial and Applied Mathematics, vol. 41, pp. 3-44, 1999, doi: 10.1137/S0036144598335107.

[32] P. K. Roy, A. N. Chatterjee, D. Greenhalgh, and Q. J. A. Khan, "Long term dynamics in a mathematical model of HIV-1 infection with delay in different variants of the basic drug therapy model," Nonlinear Analysis: Real World Applications, vol. 14, pp. 1621-1633, 2013, doi: 10.1016/j.nonrwa.2012.10.021.

[33] K. Wang, A. Fan, and A. Torres, "Global properties of an improved hepatitis B virus model," Nonlinear Analysis: Real World Applications, vol. 11, pp. 3131-3138, 2010, doi: 10.1016/j.nonrwa.2009.11.008.

[34] S. Wang and D. Zou, "Global stability of in host viral models with humoral immunity and intracellular delays," Applied Mathematical Modelling, vol. 36, no. 3, pp. 1313-1322, 2012, doi: 10.1016/j.apm.2011.07.086. 
[35] T. Wang, Z. Hu, F. Liao, and W. anbiao, "Global stability analysis for delayed virus infection model with general incidence rate and humoral immunity," Mathematics and Computers in Simulation, vol. 89, pp. 13-22, 2013, doi: 10.1016/j.matcom.2013.03.004.

[36] X. Wang and S. Liu, "A class of delayed viral models with saturation infection rate and immune response," Mathematical Methods in the Applied Sciences, vol. 36, no. 2, pp. 125-142, 2013, doi: 10.1002/mma.2576.

[37] Y. Yang, H. Wang, Z. Hu, and F. Liao, "Global stability of in-host viral model with humoral immunity and beddington-deangelis functional response," International Journal of Life Science and Medical Research, vol. 3, pp. 200-209, 2013, doi: 10.5963/LSMR0305003.

[38] S. Zeuzem, J. M. Schmidt, J. H. Lee, B. Ruster, and W. K. Roth, "Effect of interferon alfa on the dynamics of hepatitis C virus turnover," Hepatology, vol. 23, pp. 366-371, 1996, doi: 10.1002/hep.510230225.

\section{Authors' addresses}

\section{A. M. Elaiw}

King Abdulaziz University, Department of Mathematics, Faculty of Science, P.O. Box 80203, Jeddah 21589, Saudi Arabia

E-mail address: a_m_elaiweyahoo.com

\section{A. Alhejelan}

King Abdulaziz University, Department of Mathematics, Faculty of Science, P.O. Box 80203, Jeddah 21589, Saudi Arabia

Qassim University, Department of Mathematics, Faculty of Arts and Science Buraidah, P. O. Box 0001, Buridah, Saudi Arabia

E-mail address: am-math@outlook.com

\section{A. M. Shehata}

Al-Azhar University, Faculty of Science, Mathematics Department, Assiut 71511, Egypt

E-mail address: ah_moukh81@yahoo.com 